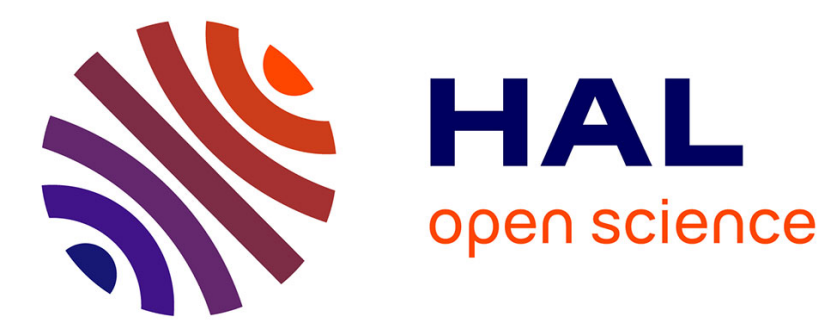

\title{
Asymptotic analysis of precoded small cell networks
}

\author{
Sreenath Ramanath, Merouane Debbah, Eitan Altman, Vinod Kumar
}

\section{To cite this version:}

Sreenath Ramanath, Merouane Debbah, Eitan Altman, Vinod Kumar. Asymptotic analysis of precoded small cell networks. IEEE International Conference on Computer Communications (INFOCOM 2010), Mar 2010, United States. 7 p. hal-00556199

\section{HAL Id: hal-00556199 \\ https://hal-centralesupelec.archives-ouvertes.fr/hal-00556199}

Submitted on 15 Jan 2011

HAL is a multi-disciplinary open access archive for the deposit and dissemination of scientific research documents, whether they are published or not. The documents may come from teaching and research institutions in France or abroad, or from public or private research centers.
L'archive ouverte pluridisciplinaire HAL, est destinée au dépôt et à la diffusion de documents scientifiques de niveau recherche, publiés ou non, émanant des établissements d'enseignement et de recherche français ou étrangers, des laboratoires publics ou privés. 


\title{
Asymptotic analysis of precoded small cell networks
}

\author{
Sreenath Ramanath ${ }^{1}$, Merouane Debbah ${ }^{2}$, Eitan Altman ${ }^{1}$, Vinod Kumar ${ }^{3}$ \\ ${ }^{1}$ INRIA, Sophia-Antipolis, France; ${ }^{2}$ SUPELEC, Paris, France; ${ }^{3}$ Alcatel-lucent Bell-labs, Paris, France;
}

\begin{abstract}
In this paper, we study precoded MIMO based small cell networks. We derive the theoretical sum-rate capacity, when multi-antenna base stations transmit precoded information to its multiple single-antenna users in the presence of intercell interference from neighboring cells. Due to an interference limited scenario, increasing the number of antennas at the base stations does not yield necessarily a linear increase of the capacity. We assess exactly the effect of multi-cell interference on the capacity gain for a given interference level. We use recent tools from random matrix theory to obtain the ergodic sum-rate capacity, as the number of antennas at the base station, number of users grow large. Simulations confirm the theoretical claims and also indicate that in most scenarios the asymptotic derivations applied to a finite number of users give good approximations of the actual ergodic sum-rate capacity.
\end{abstract}

Index Terms-Cellular networks; MIMO; Small cells; random matrix theory; linear precoding.

\section{INTRODUCTION}

Small cell based wireless networks are gaining wide popularity to provide the end user with uniform coverage, symmetry and throughput [15], [14]. Existing cellular networks like GSM and WiMAX do not achieve expected throughput to ensure seamless mobile broadband, owing to large coverage area and inability to reach indoor users. For a given radio architecture, dividing a large (macro) cell into number of small (Pico) cells is one of the most effective ways to increase both system capacity [14] and coverage to bring the user a step closer to any-place, any-time, any-device mobile broadband access.

While, dividing a macro-cell into multiple small cells enhances the capacity, the spatial dimension has been exploited in the recent past to enhance the capacity further. It is now well established that Multiple antenna at the transmitter $\left(N_{t}\right)$ and the receiver $\left(N_{r}\right)$ achieve capacity gains which grow linearly as $\min \left(N_{t}, N_{r}\right)$.

Recently, the MIMO broadcast channel [13], [6], [7], where, a multi-antenna base station, transmitting on $\mathrm{M}$ antennas to $\mathrm{K}$ single antenna users is shown to achieve capacity gains which grow linearly as $\min (M, K)$, provided the transmitter and receivers all know the channel [9]. To achieve this, several methods have been proposed among which linear precoders offer a good compromise between complexity and performance trade-off [1],[8].

Further, MIMO based systems have been studied in the framework of multi-cell networks. In a multi-cell scenario, the achievable sum-rate in the downlink, diminishes due to interference from neighboring base stations. Thus increasing the number of antennas at the base-stations does not necessarily yield a linear increase in capacity. Frequency reuse and various forms of interference co-ordination [3], [5] have been proposed to achieve linear growth in capacity.

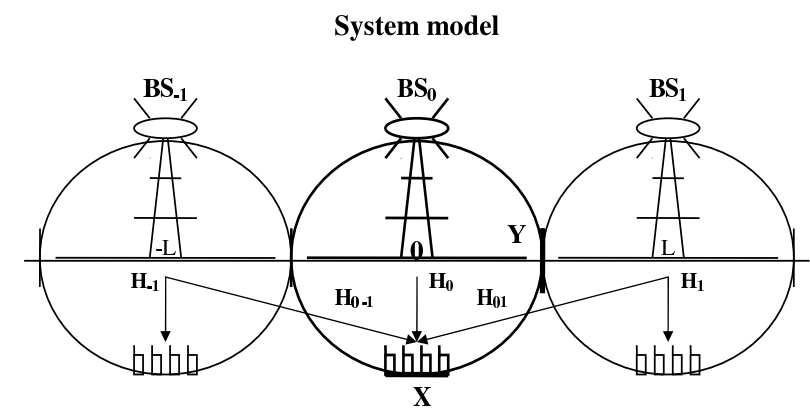

Fig. 1. System model: multi-cell network. BS with $M$ antennas, serving $K$ users. Users at $X$ experience nominal interference and users at $Y$ experience high interference

In our contribution, we want to asses exactly the effect of multi-cell interference in MIMO based small cell networks. Small cells being in close proximity experience higher levels of interference, which would bring down the capacity gains significantly. We want to study the impact of multicell interference when base-stations employ linear precoding techniques, such as channel inversion (CI) at the base station.

As mentioned before, linear precoding techniques such as channel inversion(CI) and regularized channel inversion (RCI) offer a convenient trade-off between complexity and achievable sum-rate performance [7], [8]. The behavior of CI in uncorrelated MIMO broadcast channels (MIMO-BC) has already been studied in [7], [8] for i.i.d. Gaussian channels. In particular, the authors in [7] showed that CI achieves linear growth in multiplexing-gain. Further, authors in [2], extended the case to include antenna correlations due to dense packing of the antennas at the transmitter. The analysis carried out considers single cell systems and they show that for the case of CI, the sum-rate is maximized when the number of antennas $M$ on the BS is equal to the number of users $K$.

For the multi-cell case, the problem of interference coordination in uplink has been discussed at length in [4]. In [5], authors address downlink macro-diversity in cellular systems. They study the potential benefit of base-station (BS) cooperation for downlink transmission in a modified Wynertype multicell model. They compare various precoders and obtain analytical sum rate expressions for both the fading and the non-fading case. They demonstrate via monte-carlo simulations the effectiveness of linear precoding. Authors in [13] suggests that asymptotically, equal power allocation is optimal when the channel is i.i.d. Gaussian.

In our work, we are interested in studying the impact of interference from adjacent base stations, which is more pronounced in MIMO based small cell networks on the achiev- 
able sum-rate capacity. We consider multiple-input multipleoutput (MIMO) multi-cell systems, each cell composed of a transmitter equipped with $M$ antennas and $K$ single-antenna receivers. We consider Wyner-type cellular models in our study. We neglect the effects of channel correlation due to densely packed antennas at the base-station transmitter, with a view to keep the analysis tractable.

The analytic expressions of the sum-rates for CI are derived by applying recent tools from random matrix theory (RMT). These expressions are independent of the specific channel realizations.

In our study, we find that

- The achievable sum-rate is significantly diminished by the effect of multi-cell interference in MIMO based small cell networks.

- The sum-rate capacity tends to grow sub-linearly with increasing interference.

- Also, there is an optimal number of users for a given number of antennas at the transmitter, which maximizes the sum-capacity. This depends on the interference level and the transmit power at the base-station.

The remainder of this paper is organized as follows: Section II briefly reviews various tools of random matrix theory which will be used in later derivations. Section IV introduces the multi-cell system model. In Section $\mathrm{V}$ we study channel inversion precoding. Section VI provides simulation results which are shown to corroborate the theoretical derivations. Finally in Section VII we provide our conclusions.

Notations: In the following, boldface lower-case symbols represent vectors, capital boldface characters denote matrices (I $\mathbf{I}_{N}$ is the $N \times N$ identity matrix). The Hermitian transpose is denoted $(\cdot)^{\mathrm{H}}$. The operator $\operatorname{tr}[\mathbf{X}]$ represents the trace of matrix $\mathbf{X}$. The eigenvalue distribution of an Hermitian random matrix $\mathbf{X}$ is $\mu_{\mathbf{X}}(x)$. The symbol $\mathrm{E}[\cdot]$ denotes expectation. The derivative of a function $f(x)$ of a single variable $x$ is denoted $f^{\prime}(x)$. All logarithms are base- 2 logarithms.

\section{RANDOM MATRIX THEORY TOOLS}

In this work, we are interested in the behavior of large random Hermitian matrices, and particularly in the asymptotic distribution of their eigenvalues. Specifically, the eigenvalue distribution of large Hermitian matrices converges, in many practical cases, to a definite probability distribution, hereafter called the empirical distribution of the random matrix, when the matrix dimensions grow to infinity.

A tool of particular interest in this work is the Stieltjes transform $\mathcal{S}_{\mathbf{X}}$ of a large Hermitian nonnegative definite matrix $\mathbf{X}$, defined on the half the space $\mathbb{C}-\mathbb{R}^{+}=\{z \in \mathbb{C}, \operatorname{Re}(z)<$ $0\}$, as

$$
\mathcal{S}_{\mathbf{X}}(z)=\int_{0}^{+\infty} \frac{1}{\lambda-z} \mu_{\mathbf{X}}(\lambda) \mathrm{d} \lambda
$$

where $\mu_{\mathbf{X}}$ is the empirical distribution of $\mathbf{X}$.

Couillet et al. [10] derived a fixed-point expression of the Stieltjes transform for Gaussian matrices with left- and rightside correlations in the following theorem,

Theorem 1: Let the entries of the $K \times M$ matrix $\mathbf{W}$ be i.i.d. Gaussian with zero mean and variance $1 / M$. Let $\mathbf{X}$ and $\mathbf{Q}$ be respectively $K \times K$ and $M \times M$ Hermitian nonnegative definite matrices with eigenvalue distributions $\mu_{\mathbf{X}}$ and $\mu_{\mathbf{Q}}$. We impose further that the largest eigenvalues of $\mathbf{X}$ and $\mathbf{Q}$ are bounded independently of $K, M$. Let $\mathbf{Y}$ be an $K \times K$ Hermitian matrix with the same eigenvectors as $\mathbf{X}$ and let $f$ be some function mapping the eigenvalues of $\mathbf{X}$ to those of $\mathbf{Y}$. Let $z \in \mathbb{C}^{+}=\mathbb{C} \backslash \mathbb{R}^{+}$. Then, for $M, K$ large with $K / M=1 / \beta$, the Stieltjes transform $\mathcal{S}_{\mathbf{H}}(z)$ of $\mathbf{H}=\mathbf{X}^{1 / 2} \mathbf{W Q W}^{\mathrm{H}} \mathbf{X}^{1 / 2}+\mathbf{Y}$ is approximately

$$
\mathcal{S}_{\mathbf{H}}(z)=\int\left(f(x)+x \int \frac{q \cdot \mu_{\mathbf{Q}}(q) \mathrm{d} q}{1+\frac{1}{\beta} q \mathcal{T}_{\mathbf{H}}(z)}-z\right)^{-1} \mu_{\mathbf{X}}(x) \mathrm{d} x
$$

where $\mathcal{T}_{\mathbf{H}}$ is a solution of the fixed-point equation

$\mathcal{T}_{\mathbf{H}}(z)=\int x\left(f(x)+x \int \frac{q \cdot \mu_{\mathbf{Q}}(q) \mathrm{d} q}{1+\frac{1}{\beta} q \mathcal{T}_{\mathbf{H}}(z)}-z\right)^{-1} \mu_{\mathbf{X}}(x) \mathrm{d} x$

An immediate corollary, when only right-correlation is considered, unfolds naturally as follows,

Corollary III: [11] Let the entries of the $K \times M$ matrix $\mathbf{W}$ be i.i.d. Gaussian with zero mean and variance $1 / M$. Let $\mathbf{Y}$ be an $K \times K$ Hermitian non-negative matrix with eigenvalue distribution $\mu_{\mathbf{Y}}(x)$. Moreover, let $\mathbf{Q}$ be a $M \times M$ nonnegative definite matrix with eigenvalue distribution $\mu_{\mathbf{Q}}(x)$, such that the eigenvalues of $\mathbf{Q}$ are bounded irrespectively of $M$. Then, for large $K, M$, such that $K / M=\alpha$, the Stieltjes transform on $\mathbb{C}^{+}$of the matrix

$$
\mathbf{H}=\mathbf{W Q W}^{\mathbf{H}}+\mathbf{Y}
$$

verifies approximately

$$
\mathcal{S}_{\mathbf{H}}(z)=\mathcal{S}_{\mathbf{Y}}\left(z-\int \frac{q}{1+\alpha q \mathcal{S}_{\mathbf{H}}(z)} \mu_{\mathbf{Q}}(q) \mathrm{d} q\right)
$$

\section{SySTEM MODEL AND ASSUMPTIONS}

We discuss the system model in this section. We consider a multi-cell Wyner-type model, for example as shown in figure (1). For simplicity and to be able to keep the analysis tractable, we consider a three-cell network. The cell at the center is our reference. The users in this cell experience interference from the neighboring base stations as shown. Each cell serves $K$ users from a base-station with $M$ antennas. We assume that the base station antennas are uncorrelated. The information from the base-station to its user set is precoded assuming perfect channel state information at the transmitter (CSIT). i.e, each base station knows perfectly the channel towards the users in its cell, but not the interfering channels. Users receive desired signal plus interference signals from adjacent base stations. The signal to interference noise (SINR) ratio at the user depends on its relative position with respect to its base station and adjacent base stations. We assume channel inversion (CI) precoding at the transmitter. The transmitted signals from the base stations undergo Rayleigh fading and path-loss. Further, we assume that the channel is constant for some interval long enough for the transmitter to learn and use it until it changes to a new value. We are interested in the 
behavior of the system and its sum-rate capacity. Many of our results are obtained for large limits, because the limiting results are often tractable. Nevertheless, we often consider $M, K$ small in our simulation examples. Further, all users are assumed to have the same average (but not instantaneous) received signal power, so our model assumes that the users are similar distances from the base station and are not in deep shadow fades.

\section{CHANNEL INVERSION PRECODING}

Channel inversion precoding, also referred to as zero-forcing (ZF) precoding, annihilates all the inter-user interference by performing an inversion of the channel matrix $\mathbf{H}$ at the transmitter. We begin our analysis with the single cell case, where the results are well documented ([7], [2]) and further we shall consider the multi-cell case.

\section{A. Single cell}

Without loss of generality, we consider cell 0 . The signal received by users in this cell is

$$
\mathbf{y}=\mathbf{H x}+\mathbf{n} .
$$

where, $\mathbf{H}$ is the $K \times M$ channel matrix with zero-mean unit-variance i.i.d complex Gaussian entries, $\mathbf{x}=\mathbf{G s}$ is the transmit vector obtained by linear precoding of the symbol vector $\mathbf{s}$ with the precoding matrix $\mathbf{G}$. Symbol $s_{k} \in \mathbf{s}$ for any user $k$ is complex Gaussian with zero mean and unit variance. The $M \times K$ linear precoding matrix is defined as

$$
\mathbf{G}=\alpha \mathbf{H}^{\mathrm{H}}\left(\mathbf{H} \mathbf{H}^{\mathbf{H}}\right)^{-1} .
$$

where $\alpha$ is chosen appropriately to satisfy the total transmit power constraint $\operatorname{tr}\left(E\left[\mathbf{x x}^{H}\right]\right) \leq \operatorname{tr}\left(\mathbf{G G}^{H}\right) \leq P$.

Now the received vector in Cell 0

$$
\mathbf{y}=\alpha \mathbf{s}+\mathbf{n} \text {. }
$$

The parameter $\alpha$ which satisfies the transmit power constraint and depends only on the channel realization $\mathbf{H}$ is given by

$$
\alpha^{2}=\frac{P}{\operatorname{tr}\left(\left(\mathbf{H H}^{\mathrm{H}}\right)^{-1}\right)}
$$

The SNR (signal to noise ratio) for any user $k$ is defined as

$$
\gamma_{k}=\frac{E_{s}\left[\left|\alpha s_{k}\right|^{2}\right]}{E|n|^{2}}=\frac{\alpha^{2}}{\sigma^{2}}
$$

is independent of the selected user. $\sigma^{2}$ is the noise variance.

The ergodic capacity for user $k$ is

$$
C_{k}=\log \left(1+\gamma_{k}\right) \text {. }
$$

and the sum-rate is

$$
R_{c i}=\sum_{i=1}^{K} \log \left(1+\gamma_{k}\right)
$$

\section{B. Asymptotic analysis for a single-cell}

$\alpha$ is a function of $\mathbf{H}$ and as $M, K \rightarrow \infty, \alpha$ tends to a constant. Thus the sum-rate can be written as

$$
\mathcal{R}_{\mathrm{ci}}=K \log \left(1+\gamma_{k}\right)
$$

Let us denote $\mathbf{H}^{\prime}=\frac{1}{\sqrt{M}} \mathbf{H}$ and $\mathbf{H}_{w}^{\prime}=\frac{1}{\sqrt{M}} \mathbf{H}_{w}$. It follows from (9) that

$$
\alpha^{2}=\frac{P}{\frac{1}{M} \operatorname{tr}\left(\mathbf{H}^{\prime} \mathbf{H}^{\prime \mathbf{H}}\right)^{-1}}
$$

When $M$ is large with $M / K=\beta$, the denominator of Equation (14) verifies

$$
\frac{1}{M} \operatorname{tr}\left(\mathbf{H}^{\prime} \mathbf{H}^{\prime \mathbf{H}}\right)^{-1}=\frac{1}{\beta} \int \frac{1}{\lambda} \mu_{\mathbf{H}^{\prime} \mathbf{H}^{\prime \mathbf{H}}}(\lambda) \mathrm{d} \lambda=\frac{1}{\beta} \mathcal{S}_{\mathbf{H}^{\prime} \mathbf{H}^{\prime \mathbf{H}}}(0)
$$

As a consequence, for large $(K, M)$

$$
\frac{\alpha^{2}}{\sigma^{2}} \rightarrow \frac{\rho \beta}{\mathcal{S}_{\mathbf{H}^{\prime} \mathbf{H}^{\prime \mathbf{H}}}(0)}, \text { where } \rho=P / \sigma^{2}
$$

and the sum-rate is approximately

$$
\mathcal{R}_{\mathrm{ci}}=K \log \left(1+\frac{\rho \beta}{\mathcal{S}_{\mathbf{H}^{\prime} \mathbf{H}^{\prime \mathbf{H}}}(0)}\right)
$$

According to Corollary III, $\mathcal{S}_{\mathbf{H}^{\prime} \mathbf{H}^{\prime \mathbf{H}}}(0)$ is the solution of ${ }^{1}$

$$
\begin{aligned}
\mathcal{S}_{\mathbf{H}^{\prime} \mathbf{H}^{\prime \mathbf{H}}}(0) & =\left(\int \frac{\lambda}{1+\frac{\lambda}{\beta} \mathcal{S}_{\mathbf{H}^{\prime} \mathbf{H}^{\prime \mathbf{H}}}(0)} \mu_{\Theta_{T}}(\lambda) \mathrm{d} \lambda\right)^{-1} \\
& =\left(\int \frac{\lambda \delta(\lambda-1)}{1+\frac{\lambda}{\beta} \mathcal{S}_{\mathbf{H}^{\prime} \mathbf{H}^{\prime \mathbf{H}}}(0)}\right)^{-1} \\
& =\left(1+\frac{\mathcal{S}_{\mathbf{H}^{\prime} \mathbf{H}^{\prime} \mathbf{H}}(0)}{\beta}\right)
\end{aligned}
$$

Solving for $\mathcal{S}_{\mathbf{H}^{\prime} \mathbf{H}^{\prime \mathbf{H}}}(0)$ yields,

$$
\mathcal{S}_{\mathbf{H}^{\prime} \mathbf{H}^{\prime \mathbf{H}}}(0)=\frac{\beta}{(\beta-1)}
$$

and the sum-rate is re-written as

$$
\mathcal{R}_{\mathrm{ci}}=K \log (1+\rho(\beta-1)) \text { for } \beta \geq 1
$$

That is,

$$
\frac{\mathcal{R}_{\mathrm{ci}}}{M}=\frac{1}{\beta} \log (1+\rho(\beta-1))
$$

defines the rate per antenna.

As $\beta \rightarrow 1, \mathcal{R}_{\text {ci }} / M \rightarrow 0$, which implies that the sum rate of channel inversion does not increase linearly with $M$ (or $K$ )

\section{Optimizer $\beta^{*}$ for the single cell}

Following [7] we now look for a value $\beta^{\star}$ of the ratio $M / K$ such that, for a fixed number of transmit antennas $M$, the

\footnotetext{
${ }^{1}$ it is important to note here that we slightly misapply Corollary III since the result is only proven valid outside for any $z>0$.
} 
sum-rate $\mathcal{R}_{\mathrm{ci}}(\beta)$ is maximized. By differentiating eqn (21) with respect to $\beta$ and setting the derivative to zero, $\beta^{*}$ is the solution of the implicit equation

$$
\rho \beta^{*}=\left(1+\rho\left(\beta^{*}-1\right)\right) \log \left(1+\rho\left(\beta^{*}-1\right)\right)
$$

\section{Multi-cell}

In this section, we study the effect of multi-cell interference. Without loss of generality, we consider users in Cell 0 affected by interference from adjacent base-stations. We consider a 3cell Wyner-type model as shown in figure 1 . Cell $C_{0}$ is at the center. Adjacent cells are designated Cell $C_{1}$ and Cell $C_{-1}$.

Following our analysis of the single cell case, the received vector for users of cell $C_{0}$, is

$\mathbf{y}=\mathbf{H}_{0} \mathbf{G}_{0} \mathbf{s}_{0}+\sqrt{\gamma} \mathbf{H}_{01} \mathbf{G}_{1} \mathbf{s}_{1}+\sqrt{\gamma} \mathbf{H}_{0-1} \mathbf{G}_{-1} \mathbf{s}_{-1}+\mathbf{n}$.

As before, $\mathbf{H}_{0}$ is the channel matrix from base station in cell $C_{0}$ to its users. $\mathbf{H}_{01}$ and $\mathbf{H}_{0-1}$ are interfering channels from cell $C_{1}$ and $C_{-1}$, respectively. $\mathbf{G}_{1}$ and $\mathbf{G}_{-1}$ are precoding matrices for users in cell $C_{1}$ and $C_{-1}$, respectively. $\gamma$ is the signal (interference) attenuation.

As stated earlier, all users in cell $C_{0}$ are assumed to have the same average received signal power, so our model assumes that the users are similar distances from the base station and are not in deep shadow fades.

The precoding matrices in cell $i$ can be written as

$$
\mathbf{G}_{i}=\alpha_{i} \mathbf{H}_{i}^{\mathrm{H}}\left(\mathbf{H}_{i} \mathbf{H}_{i}^{\mathrm{H}}\right)^{-1}
$$

The ergodic capacity for user $K$ is expressed as

$$
C_{k}=\log \left(1+\frac{\alpha_{0}^{2}}{E\left[\left|n_{k}^{\prime}\right|^{2}\right]}\right)
$$

Where, $n_{k}$ is the $k-t h$ element of the covariance matrix $n$. The expectation of this matrix can be written as

$$
\begin{aligned}
E\left[n^{\prime} n^{\prime} \mathbf{H}\right] & =\gamma \mathbf{H}_{01} \mathbf{G}_{1} \mathbf{G}_{1}^{\mathrm{H}} \mathbf{H}_{01}^{\mathrm{H}} \\
& +\gamma \mathbf{H}_{0-1} \mathbf{G}_{-1} \mathbf{G}_{-1}^{\mathrm{H}} \mathbf{H}_{0-1}^{\mathrm{H}}+\sigma^{2} I
\end{aligned}
$$

Expanding and simplifying,

$$
\begin{aligned}
E\left[n^{\prime} n^{\prime}{ }^{H}\right] & =\gamma \alpha_{1}^{2} \mathbf{H}_{01} \mathbf{H}_{1}^{\mathrm{H}}\left(\mathbf{H}_{1} \mathbf{H}_{1}^{\mathrm{H}}\right)^{-2} \mathbf{H}_{1} \mathbf{H}_{01}^{\mathrm{H}} \\
& +\gamma \alpha_{-1}^{2} \mathbf{H}_{0-1} \mathbf{H}_{-1}^{\mathrm{H}}\left(\mathbf{H}_{-1} \mathbf{H}_{-1}^{\mathrm{H}}\right)^{-2} \mathbf{H}_{-1} \mathbf{H}_{0-1}^{\mathrm{H}} \\
& +\sigma^{2} I \\
E\left[n^{\prime} n^{\prime}{ }^{H}\right] & =\gamma \alpha_{1}^{2} \mathbf{H}_{01} \mathbf{H}_{1}^{\mathrm{H}}\left(\mathbf{H}_{1} \mathbf{H}_{1}^{\mathrm{H}}\right)^{-2} \mathbf{H}_{1} \mathbf{H}_{01}^{\mathrm{H}} \\
& +\gamma \alpha_{-1}^{2} \mathbf{H}_{0-1} \mathbf{H}_{-1}^{\mathrm{H}}\left(\mathbf{H}_{-1} \mathbf{H}_{-1}^{\mathrm{H}}\right)^{-2} \mathbf{H}_{-1} \mathbf{H}_{0-1}^{\mathrm{H}} \\
& +\sigma^{2} I
\end{aligned}
$$

Since,

$$
E\left[\left|n_{1}^{\prime}\right|^{2}\right]=E\left[\left|n_{2}^{\prime}\right|^{2}\right] \ldots=E\left[\left|n_{k}^{\prime}\right|^{2}\right]
$$

We can write,

$$
\begin{aligned}
& E\left[\left|n_{k}^{\prime}\right|^{2}\right] \rightarrow \frac{1}{K} \sum_{k=1}^{K} E\left[\left|n_{i}^{\prime}\right|^{2}\right] \\
&= \frac{1}{K} \operatorname{tr}\left(E\left[n^{\prime} n^{\prime}{ }^{H}\right]\right) \\
& E\left[\left|n_{k}^{\prime}\right|^{2}\right] \rightarrow \frac{1}{K} \operatorname{tr}\left(\gamma \alpha_{1}^{2} \mathbf{H}_{01} \mathbf{H}_{1}^{\mathrm{H}}\left(\mathbf{H}_{1} \mathbf{H}_{1}^{\mathrm{H}}\right)^{-2} \mathbf{H}_{1} \mathbf{H}_{01}^{\mathrm{H}}\right. \\
&\left.+\gamma \alpha_{-1}^{2} \mathbf{H}_{0-1} \mathbf{H}_{-1}^{\mathrm{H}}\left(\mathbf{H}_{-1} \mathbf{H}_{-1}^{\mathrm{H}}\right)^{-2} \mathbf{H}_{-1} \mathbf{H}_{0-1}^{\mathrm{H}}\right) \\
&+\sigma^{2} I
\end{aligned}
$$

\section{E. Asymptotic analysis for the multi-cell}

We can show that

$\frac{1}{K} \operatorname{tr}\left(\mathbf{H}_{01} \mathbf{H}_{1}^{\mathrm{H}}\left(\mathbf{H}_{1} \mathbf{H}_{1}^{\mathrm{H}}\right)^{-2} \mathbf{H}_{1} \mathbf{H}_{01}^{\mathrm{H}}\right) \rightarrow \frac{1}{\beta}$ as $K, M \rightarrow \infty$

Now the expectation reduces to,

$$
E\left[\left|n_{k}^{\prime}\right|^{2}\right] \rightarrow \alpha_{1}^{2} \gamma \frac{1}{\beta}+\alpha_{-1}^{2} \gamma \frac{1}{\beta}+\sigma^{2}
$$

And hence, the sum-rate is

$$
\mathcal{R}_{\mathrm{ci}}=K \log \left(1+\frac{\alpha_{0}^{2} \beta}{\alpha_{1}^{2} \gamma+\alpha_{-1}^{2} \gamma+\sigma^{2} \beta}\right)
$$

Following 16, for large $(K, M)$,

$$
\frac{\alpha_{0}^{2}}{\sigma^{2}}=\frac{\alpha_{1}^{2}}{\sigma^{2}}=\frac{\alpha_{-1}^{2}}{\sigma^{2}} \rightarrow \frac{\rho \beta}{\mathcal{S}_{\mathbf{H}^{\prime} \mathbf{H}^{\prime \mathbf{H}}}(0)}, \text { where } \rho=P / \sigma^{2}
$$

Thus the above sum-rate expression can be simplified as

$$
\mathcal{R}_{\mathrm{ci}}=K \log \left(1+\frac{\rho \beta}{\mathcal{S}_{\mathbf{H}^{\prime} \mathbf{H}^{\prime \mathbf{H}}}(0)+2 \gamma \rho}\right)
$$

Substituting for $\mathcal{S}_{\mathbf{H}^{\prime} \mathbf{H}^{\prime \mathbf{H}}}(0)$,

$$
\mathcal{R}_{\mathrm{ci}}=K \log \left(1+\frac{\rho \beta(\beta-1)}{(\beta+2 \gamma \rho(\beta-1))}\right)
$$

Re-writing,

$$
\frac{\mathcal{R}_{\mathrm{ci}}}{M}=\frac{1}{\beta} \log \left(1+\frac{\rho \beta(\beta-1)}{(\beta+2 \gamma \rho(\beta-1))}\right)
$$

We observe that when $\gamma=0$, that is when there is no interference, the capacity formula is that of the single-cell case.

As $\beta \rightarrow 1, \mathcal{R}_{\text {ci }} / M \rightarrow 0$, which implies that the sum rate of channel inversion does not increase linearly with $M$ (or $K$ )

\section{F. Optimizer $\beta^{*}$ for the multi-cell}

Following on similar lines of the single-cell case, we now look for a value $\beta^{\star}$ of the ratio $M / K$ such that, for a fixed number of transmit antennas $M$, the sum-rate $\mathcal{R}_{\mathrm{ci}}(\beta)$ is maximized. By differentiating eqn (38) with respect to $\beta$ and setting the derivative to zero, $\beta^{*}$ is the solution of the 


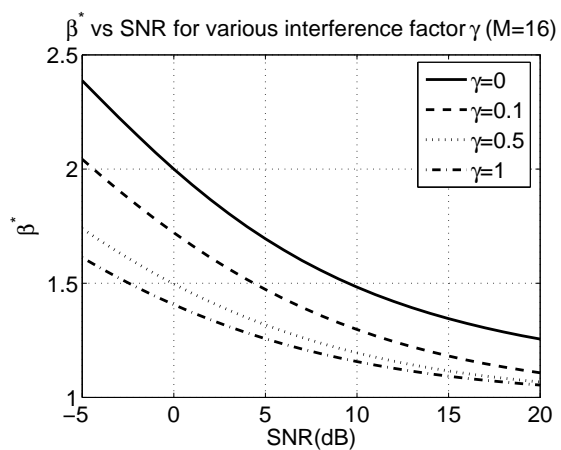

Fig. 2. $\beta^{*}$ vs SNR for various interference factors $(M=16)$

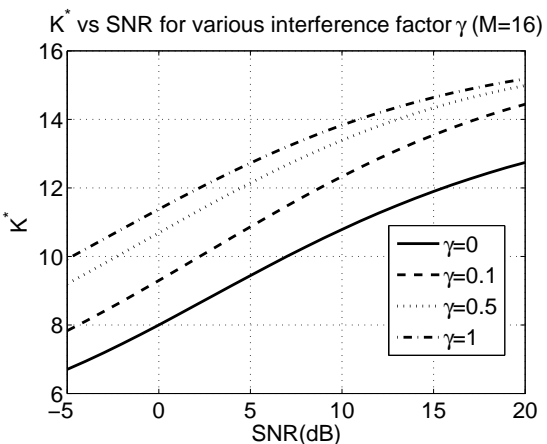

Fig. 3. $K^{*}$ vs SNR for various interference factors $(M=16)$

implicit equation

$$
\begin{array}{r}
\rho \beta^{*}=\frac{\left[\beta^{*}+2 \gamma \rho\left(\beta^{*}-1\right)\right]\left[\beta^{*}+\rho \beta^{*}\left(\beta^{*}-1\right)+2 \gamma \rho\left(\beta^{*}-1\right)\right]}{\left(\left(\beta^{*}\right)^{2}+2 \gamma \rho\left(\beta^{*}-1\right)^{2}\right)} \\
\log \left[1+\frac{\rho \beta^{*}\left(\beta^{*}-1\right)}{\left[\beta^{*}+2 \gamma \rho\left(\beta^{*}-1\right)\right]}\right]
\end{array}
$$

One can observe that by setting $\gamma=0$, we fall back to the implicit equation (22) of the single cell case.

\section{G. Some observations:}

Following our single cell and multi-cell analysis, we plot in figure 2 , the optimal $\beta$, i.e, $\beta^{*}$ (refer equation 39), which maximizes the sum rate and in figure 3 the corresponding optimal number of users $K^{*}=M / \beta^{*}$ for $\mathrm{M}=16$ and different SNR. We observe that,

1). With increasing SNR more and more users should be served to maximize the sum rate.

2). Also, the number of users required to maximize the sum rate tends to increase with an increase in the interference factor $\gamma$.

Next, we plot the optimal sum rate (refer equation 38), i.e, the sum rate achieved when $\beta=\beta^{*}$ in figure 4 . We compare this for example with $\beta=2$, shown in figure 5 . We obtain the sum-rate by computing the sum-rate per user in the asymptotic regime and then multiplying this with a finite number of antennas $M$ at the BS. For this example we have used $M=2$.

There are some interesting observations here:

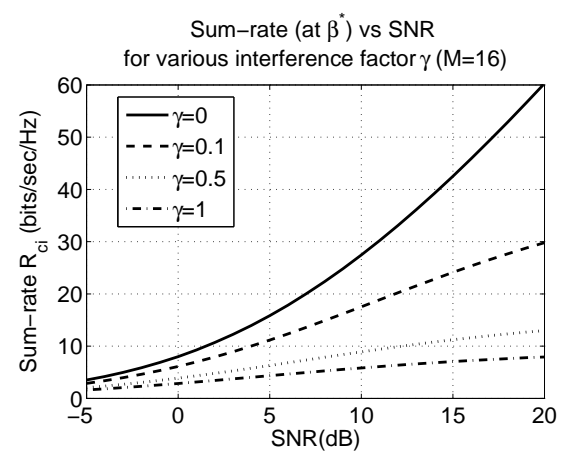

Fig. 4. Sum rate at $\beta^{*}$ for various interference factors $(M=16)$ Sum-rate at $(\beta=2)$ vs SNR

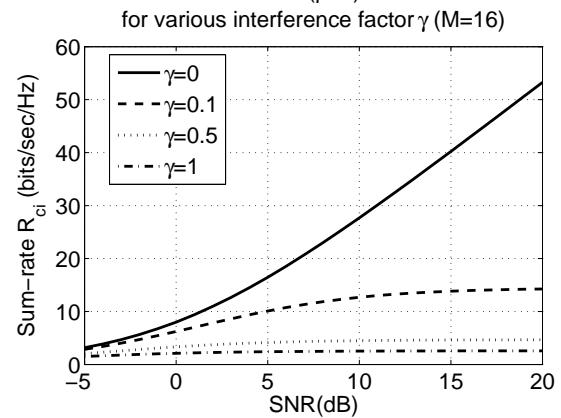

Fig. 5. Sum rate at $\beta=2$ for various interference factors $(M=16)$

1). The capacity tends to increase at a constant rate when $\beta=\beta^{*}$, irrespective of whether there is interference or not, albeit at a very slow rate with high interference.

2 ). We also see that the sum-rate tends to saturate if we deviate from the optimal $\beta$, i.e, $\beta^{*}$.

(39). The saturation occurs sooner when the interference is higher.

\section{H. Single cell and multi-cell with power constraint}

The sum-rate per antenna in the case of single-cell is given by

$$
\frac{R_{c i}}{M}=\frac{1}{\beta} \log \left(1+p_{i} \rho(\beta-1)\right)
$$

Where $p_{i}=P / M$.

For the multi-cell case, we re-write the ergodic capacity eqn 25 for user $k$ when base-stations use equal power constraint $p_{0}=P_{0} / M$ as

$$
C_{k}=\log \left(1+\frac{\alpha_{0}^{2} p_{0}}{E\left[\left|n_{k}^{\prime}\right|^{2}\right]}\right)
$$

Where,

$$
\begin{aligned}
E\left[n^{\prime} n^{\prime}{ }^{H}\right] & =\gamma \mathbf{H}_{01} \mathbf{G}_{1} \mathbf{P}_{1} \mathbf{G}_{1}^{\mathrm{H}} \mathbf{H}_{01}^{\mathrm{H}} \\
& +\gamma \mathbf{H}_{0-1} \mathbf{G}_{-1} \mathbf{P}_{-1} \mathbf{G}_{-1}^{\mathrm{H}} \mathbf{H}_{0-1}^{\mathrm{H}} \\
& +\sigma^{2} I
\end{aligned}
$$

After suitable simplification similar to the multi-cell analysis in the previous section, we can re-write the above expression 
as

$$
\begin{array}{r}
E\left[\left|n_{k}^{\prime}\right|^{2}\right] \rightarrow \frac{1}{K} \operatorname{tr}\left(\mathbf{H}_{01} \mathbf{H}_{1}^{\mathrm{H}}\left(\mathbf{H}_{1} \mathbf{H}_{1}^{\mathrm{H}}\right) \mathbf{P}_{1}\left(\mathbf{H}_{1} \mathbf{H}_{1}^{\mathrm{H}}\right) \mathbf{H}_{1} \mathbf{H}_{1}^{\mathrm{H}}\right. \\
\left.+\mathbf{H}_{0-1} \mathbf{H}-1^{\mathrm{H}}\left(\mathbf{H}-1 \mathbf{H}_{-1}^{\mathrm{H}}\right) \mathbf{P}_{-1}\left(\mathbf{H}_{-1} \mathbf{H}_{-1}^{\mathrm{H}}\right) \mathbf{H}_{-1} \mathbf{H}_{-1}^{\mathrm{H}}\right) \\
+\sigma^{2} I
\end{array}
$$

It is shown that in the asymptotic regime

$$
\frac{1}{K} \operatorname{tr}\left(\mathbf{H}_{01} \mathbf{H}_{1}^{\mathrm{H}}\left(\mathbf{H}_{1} \mathbf{H}_{1}^{\mathrm{H}}\right) \mathbf{P}_{1}\left(\mathbf{H}_{1} \mathbf{H}_{1}^{\mathrm{H}}\right) \mathbf{H}_{1} \mathbf{H}_{1}^{\mathrm{H}}\right) \rightarrow \frac{1}{\beta} \operatorname{tr}\left(P_{1}\right)
$$

Therefore, the expectation can be written as,

$$
E\left[\left|n_{k}^{\prime}\right|^{2}\right]=\gamma \alpha_{1}^{2} \frac{1}{\beta} \operatorname{tr}\left(P_{1}\right)+\gamma \alpha_{-1}^{2} \frac{1}{\beta} \operatorname{tr}\left(P_{-1}\right)+\sigma^{2} I .
$$

And hence, the sum-rate is

$$
K \log \left(1+\frac{\alpha_{0}^{2} \beta p_{0}}{\mathcal{R}_{\mathrm{ci}}}=\right.
$$

Following 16, for large $(K, M)$,

$$
\frac{\alpha_{0}^{2}}{\sigma^{2}}=\frac{\alpha_{1}^{2}}{\sigma^{2}}=\frac{\alpha_{-1}^{2}}{\sigma^{2}} \rightarrow \frac{\rho \beta}{\mathcal{S}_{\mathbf{H}^{\prime} \mathbf{H}^{\prime \mathbf{H}}}(0)}, \text { where } \rho=P / \sigma^{2}
$$

Now, the sum-rate can be written as,

$$
\begin{array}{r}
\frac{\mathcal{R}_{\mathrm{ci}}}{M}= \\
\frac{1}{\beta} \log \left(1+\frac{\rho \beta(\beta-1) p_{0}}{\left(\beta+\gamma \rho(\beta-1) \operatorname{tr}\left(P_{1}\right)+\gamma \rho(\beta-1) \operatorname{tr}\left(P_{-1}\right)\right)}\right)
\end{array}
$$

Thus, one can conclude that in the equal power regime, if some of the users in the adjacent base stations are not being serviced, i.e, their respective antenna at the transmitter is switched-off, for example, $\operatorname{tr}\left(P_{1}\right)<P_{1}$, the interference comes down and hence the capacity scales up.

\section{Simulations RESUlts:}

In this section we evaluate by simulation how interference from neighboring base stations impacts the behavior of the sum-rate of linearly precoded MIMO small cell networks when the antenna array at the transmitter is dense. We compare numerical results obtained by Monte-Carlo simulations with our previously derived asymptotic expressions for finite $(K, M)$. In particular, we have the following cases.

i.) We fix the SNR $(\rho=20 \mathrm{~dB})$ and calculate rate achieved per antenna as we vary $\beta=M / K$ (refer equation 38). We plot this in figure 6 for various interference factors $\gamma$. We observe that the rate per antenna is maximized for a certain $\beta=\beta^{*}$. This matches with the $\beta^{*}$ computed by solving the implicit equation 39. It is also interesting to observe that $\beta^{*}$ decreases with increasing interference. Also, beyond $\beta^{*}$, the capacity growth is not in proportion to the growth in number of antennas at the base station $M$

ii.) We fix the SNR $(\rho=20 \mathrm{~dB})$ and the ratio $M / K=$ $\beta=2$. We compute the rate achieved per antenna as we vary the interference factor $\gamma$. We compare asymptotic results via monte-carlo simulations. We plot this in figure 7. We observe

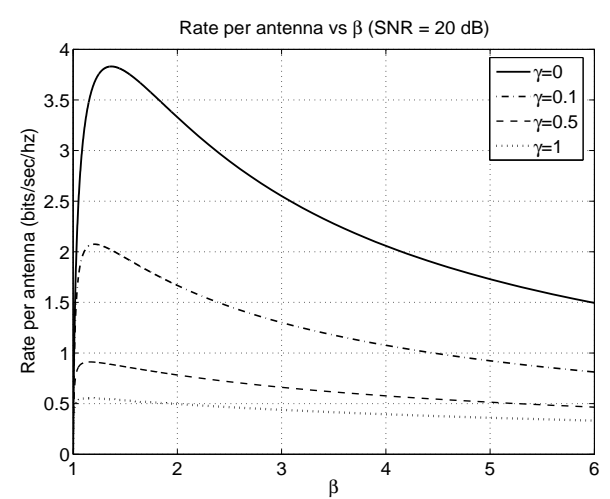

Fig. 6. Rate per antenna vs $\beta$ at SNR of $20 \mathrm{~dB}$ for various interference factors $\gamma$

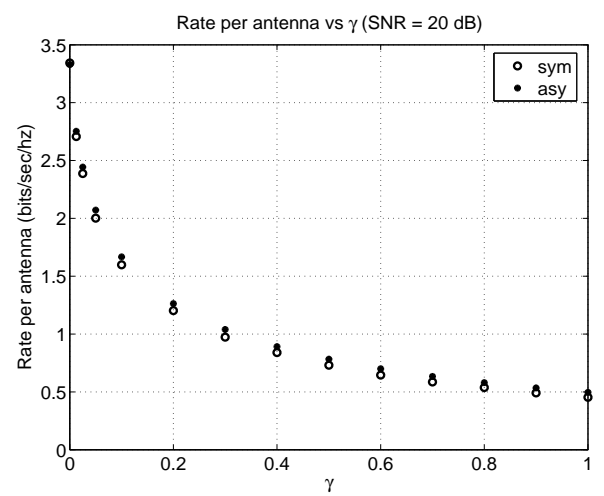

Fig. 7. Rate per antenna vs $\gamma$, when, $\beta=2$, SNR $\rho=20 \mathrm{~dB}$ for various interference factors $\gamma$

that the achievable rate is very sensitive to interference. The drop in rate is very steep in the beginning and tends to normalize for higher interference. This seems to indicate that the high amount of interference envisaged in small cells might not be as harmful. Many of the proposed interference management and co-ordination schemes might work well even in the case of small cells.

iii.) Next we show how the sum-rate increases with increasing number of base-station antennas $M$ at $\operatorname{SNR}(\rho=0,20 d B)$ for various interference factors $\gamma$, when $\beta=2$. We compute the rate per antenna from equation 38 for the asymptotic part to compare it with monte-carlo simulations. The observations are plotted in figures 8,9 . We observe that the increase in sum-rate is linear when interference is nil. The increase is sub-linear for other interference factors. Since the number of antennas at the base station and number of users are increasing simultaneously, the capacity is expected to grow in proportion to $\min (M, K)$, scaled by a factor, that depends on the interference factor $\gamma$ and the SNR $\rho$.

In all the cases, we observe that in all simulations the asymptotic results closely match the numerical results even for small values of $(K, M)$.

\section{CONCLUSIONS}

We looked at the problem of inter-cell interference in MIMO based small cell networks. We started our analysis with a 


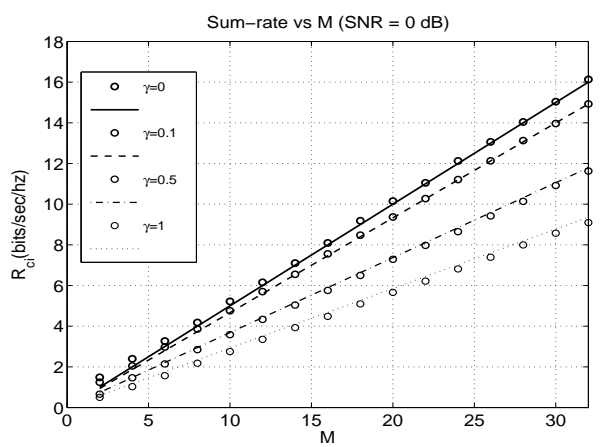

Fig. 8. Sum rate per antenna as a function of $M$ for $\beta^{*}$ at SNR of $0 \mathrm{~dB}$ for various interference factors

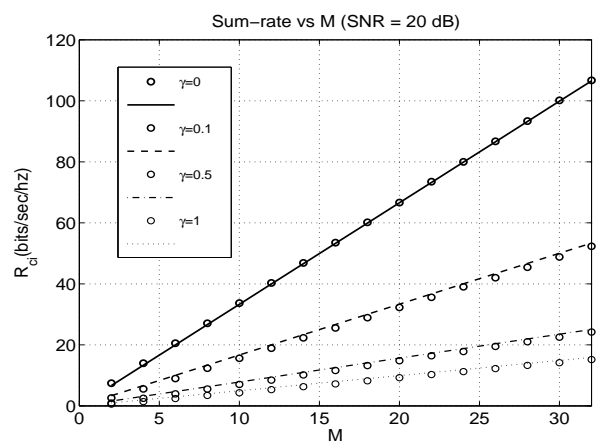

Fig. 9. Sum rate per antenna as a function of $M$ for $\beta^{*}$ at SNR of $20 \mathrm{~dB}$ for various interference factors

single cell, where multi-antenna base station employ channel inversion precoding to communicate with multiple singleantenna users. We extended the case to multi-cell scenario, using a simple wyner-type model. We derived the sum-rate capacity in the asymptotic regime, i.e, when the number of antennas at the base station and number of user grow large, but, with a fixed ratio. We used recent tools from random matrix theory, which have proven to give reliable results even when the quantities involved are practical and finite. We further derived $\beta^{*}$, the ratio of number of transmit antennas to users, which maximizes the achievable sum-rate. The asymptotic analysis was validated with monte-carlo simulations in the finite regime.

We conclude that the achievable sum-rate is significantly diminished by the effect of multi-cell interference in MIMO based small cell networks. The sum-rate capacity tends to grow sub-linearly with increasing interference. Also, there is an optimal number of users for a given number of antennas at the transmitter, which maximizes the sum-capacity. This depends on the interference level and the transmit power at the base-station. For a given number of transmit antenna, moving away from the optimal, $\beta^{*}$, tends to saturate the capacity growth at high SNR. The saturation occurs sooner with higher interference.

\section{ACKNOWLEDGMENT}

This work was done in the framework of the INRIA and Alcatel-Lucent Bell Labs Joint Research Lab on Self
Organized Networks. The work of Merouane Debbah is done within the Alcatel-lucent chair on Flexible Radio.

\section{REFERENCES}

[1] Mai Vu and Arogyaswami Paulraj, "MIMO wireless linear precoding”, IEEE Signal Processing Magazine, Sep 2007.

[2] Romain Couillet, Sebastian Wagner and Merouane Debbah, "Asymptotic Analysis of Linear Precoding Techniques in Correlated MultiAntenna Broadcast Channels", Submitted to IEEE Transactions on Information Theory.

[3] Oren Somekh, Member, Benjamin M. Zaidel and Shlomo Shamai (Shitz), "Sum Rate Characterization of Joint Multiple Cell-Site Processing", IEEE Transactions on Information Theory, Vol. 53, No. 12, Dec 2007

[4] Sivarama Venkatesan, Angel Lozano, Reinaldo Valenzuela, "Network MIMO: Overcoming Intercell Interference in Indoor Wireless Systems", Asilomar 2007

[5] Sheng Jing, David N. C. Tse, Joseph B. Soriaga I, Jilei Hou, John E. Smeet and Roberto Padovani, "Downlink Macro-Diversity in Cellular Networks", ISIT 2007

[6] H. Weingarten, Y. Steinberg, and S. Shamai, The Capacity Region of the Gaussian Multiple-Input Multiple-Output Broadcast Channel, Information Theory, IEEE Transactions on, vol. 52, no. 9, pp. 39363964, 2006.

[7] B. Hochwald and S. Vishwanath, Space-Time Multiple Access: Linear Growth in the Sum Rate, in Proceedings of the annual allerton conference on communication control and computing, vol. 40, no. 1. The University; 1998, 2002, pp. 387396

[8] C. Peel, B. Hochwald, and A. Swindlehurst, A Vector-Perturbation Technique for Near-Capacity Multiantenna Multiuser CommunicationPart I: Channel Inversion and Regularization, Communications, IEEE Transactions on, vol. 53, no. 1, pp. 195202, 2005.

[9] N. Jindal and A. Goldsmith, Dirty-paper coding versus TDMA for MIMO Broadcast channels, Information Theory, IEEE Transactions on, vol. 51, no. 5, pp. 17831794, 2005

[10] R. Couillet, M. Debbah, and J. Silverstain, A deterministic equivalent approach for the capacity analysis of multi-user MIMO channels, submitted to IEEE Trans. on Information Theory.

[11] J. Silverstein and Z. Bai, On the Empirical Distribution of Eigenvalues of a Class of Large Dimensional Random Matrices, Journal of Multivariate Analysis, vol. 54, pp. 175175, 1995.

[12] M. Debbah and R. Muller, MIMO channel modeling and the principle of maximum entropy, Information Theory, IEEE Transactions on, vol. 51, no. 5, pp. 16671690, 2005.

[13] H. Viswanathan and S. Venkatesan, Asymptotics Of Sum Rate for Dirty Paper Coding and Beamforming In Multiple-Antenna Broadcast Channels, Allerton 1998

[14] Gregory Davi, "Using picocells to build high-throughput 802.11 networks" RF Design, Jul 2004.

[15] "Picocell Mesh: Bringing Low-Cost Coverage, Capacity and Symmetry to Mobile WiMAX", A Tropos Networks white paper 